\title{
Colección selectiva como instrumento de conservación ambiental y fuente de ingresos
}

\author{
Selective collection as environmental preservation instrument and income \\ source
}

\section{Coleta seletiva como instrumento de preservação ambiental e fonte de renda}

\author{
Everaldo Oliveira de Souza' \\ Danila Fernanda Rodrigues Frias"
}

\begin{abstract}
Resumen
La acumulación de residuos sólidos causa deterioro ambiental e implicaciones para la calidad de vida, por lo que la recolección selectiva surge como una alternativa para reducir este impacto. El objetivo de este estudio fue evaluar los perfiles socioeconómicos, demográficos y laborales de los recicladores en llha Solteira, São Paulo y analizar la importancia de esta práctica para la subsistencia y la preservación del medio ambiente. Para ello, se entrevistó a 22 recicladores y se recabó información del Ayuntamiento. Entre los encuestados, $72.7 \%$ eran hombres, $54.5 \%$ casados, $54.6 \%$ vivían favorablemente, $40.9 \%$ reportaron estar en la actividad por más de 5 años, $90.9 \%$ se debieron al desempleo y $66,3 \%$ declararon que consideran la práctica de la preparación. Muy importante para mantener la ciudad más limpia. Con respecto a los datos obtenidos del Ayuntamiento, se encontró que, en su sitio web, se divulga una serie de información sobre cómo los ciudadanos pueden ayudar en la recolección selectiva y el control de los desechos generados. La profesión de carroñero demostró ser muy importante porque muchas personas usan esta práctica como ocupación y fuente de ingresos para mantener a sus familias. La colección debe realizarse de manera digna y tener el debido reconocimiento y reconocimiento de la sociedad.
\end{abstract}

Palabras clave: Impacto ambiental; Reciclaje; Salud pública

\begin{abstract}
The accumulation of solid waste causes environmental deterioration and implications for quality of life, so selective collection emerges as an alternative to reduce this impact. The objective of this study was to evaluate the socioeconomic, demographic and work profiles of waste pickers in Ilha Solteira, São Paulo and to analyze the importance of this practice for subsistence and environmental preservation. For this, 22 waste pickers were interviewed and information was collected from the City Hall. Among the respondents, $72.7 \%$ were male, $54.5 \%$ married, $54.6 \%$ lived favorably, $40.9 \%$ reported being in the activity for more than 5 years, $90.9 \%$ are due to unemployment and $66,3 \%$ stated that they consider the practice of grooming very important for maintaining
\end{abstract}


the city cleaner. Regarding the data obtained from the City Hall, it was found that, on its website, disclosure of a series of information on how citizens can help in the selective collection and control of waste generated. The profession of scavenger proved to be very important because many people use this practice as an occupation and source of income to support their families. The collection must be performed in a dignified manner and have due appreciation and recognition by society.

Keywords: Environmental Impact; Recycling; Public health

\section{Resumo}

O acúmulo de resíduos sólidos causa deterioração ambiental e implicações para qualidade de vida, por isso a coleta seletiva surge como alternativa para diminuir este impacto. $O$ objetivo neste trabalho foi avaliar os perfis socioeconômicos, demográficos e de trabalho dos catadores de llha Solteira, São Paulo e analisar a importância desta prática para subsistência e preservação ambiental. Para isso foram entrevistados 22 catadores e realizado levantamento de informações junto a Prefeitura Municipal. Dentre os entrevistados $72,7 \%$ eram do sexo masculino, 54,5\% casados, 54,6\% moravam de favor, 40,9\% relataram estar na atividade a mais de 5 anos, $90,9 \%$ estão devido ao desemprego e $66,3 \%$ declararam que consideram a prática de catação muito importante para manutenção da cidade mais limpa. Com relação aos dados obtidos na Prefeitura verificou-se que, em seu website, são divulgadas informações de como os cidadãos podem ajudar na coleta seletiva e no controle do resíduo gerado. A profissão de catador demonstrou ser muito importante pois várias pessoas utilizam esta prática como ocupação e fonte de renda para sustento de sua família. A catação deve ser realizada de forma digna e possuir a devida valorização e reconhecimento pela sociedade.

Palavras-chave: Impacto Ambiental; Reciclagem; Saúde Pública 


\section{Introduccion}

En Brasil y en todo el mundo, el tema de la sostenibilidad y el desarrollo sostenible ocupa un lugar destacado en los debates. Una de las principales razones de esta discusión es el rápido crecimiento de la población que trae consigo nuevas necesidades y nuevos niveles de consumo de productos (LIMA et al, 2007).

Para mantener el ritmo de las necesidades globales, las industrias tienden a producir más innovando y creando productos, la mayoría de ellos sin características biodegradables, lo que resulta en miles de millones de envases que se convierten en residuos en manos del consumidor final (LIMA et al, 2007 )

Hoy se habla mucho sobre la reutilización, recuperación y transformación de materiales reciclables, precisamente como una alternativa al consumo desenfrenado y como una herramienta de colaboración con la que se busca el desarrollo sostenible. La idea del desarrollo sostenible en todo el mundo surgió a mediados de 1987 a través del Informe Brundtland, preparado por la Comisión Mundial de Medio Ambiente y Desarrollo, establecido en 1983 por la Asamblea de las Naciones Unidas (MARODIN; MORAIS, 2004).

En Brasil, la idea de desarrollo sostenible ganó fuerza a través de la Conferencia ECO de 1992, celebrada por las Naciones Unidas en Río de Janeiro, en 1992. Esta Conferencia, también conocida como la Cumbre de la Tierra, reunió a más de 100 jefes de estado. para discutir formas de desarrollo sostenible (LAGO, 2007).

Después de esta Conferencia, las autoridades de todo el mundo comenzaron a mirar a los problemas ambientales con mayor intensidad, así como a abordarlos como un factor principal en la supervivencia del Planeta Tierra.

Otro paso importante en la búsqueda del desarrollo sostenible fue la formalización del Protocolo de Kioto, que tuvo lugar en Japón en 1997. El objetivo principal de este protocolo era reducir las emisiones de gases de efecto invernadero para mitigar los efectos del calentamiento global. Fijó objetivos a cumplir por cada país, de acuerdo con sus condiciones geográficas, económicas y políticas (WEYERMÜLLER, 2010). 
Hoy en día, la gran acumulación de desechos es un grave problema contemporáneo que lleva a la humanidad a serios riesgos sociales y para la salud, ya que todo lo que se produce es generalmente de menor durabilidad que en el pasado, y debe reemplazarse a corto plazo. Conocidos como "desechables", estos productos se usan solo una vez o por un corto tiempo y luego se descartan, como ejemplos de pañales, botellas de vidrio, aluminio, pajitas, tazas, bolsas y envases en general, además del gran volumen. de artículos utilizados diariamente (SALDIVA, 2010).

Según los datos de la Encuesta Nacional de Muestra de Hogares (PNAD), el número de hogares en Brasil creció de 69.5 millones en 2017 a 71.0 millones en 2018, lo que representa un aumento del 2.2\%. En comparación con 2016, el aumento fue del 3,1\% (2,1 millones de unidades) (IBGE, 2019).

En el país, en 2018, el $83.0 \%$ de los hogares tenía acceso a la recolección directa de desechos y el $8.1 \%$ recolectado a través de un contenedor de basura, mientras que el $8.9 \%$ quemó los desechos en la propiedad o le dio otro destino, como depositar en valones, por ejemplo. Esto representa un contingente de 20,1 millones de personas sin acceso a algún tipo de recolección de residuos. Entre las Grandes Regiones, el Nordeste tenía el mayor contingente de residentes en estas condiciones, con 10.5 millones, seguido por el Norte, con 3.8 millones. Más de una cuarta parte de los hogares quemaron residuos en propiedades en Maranhão (27.5\%) y Piauí (26.5\%) (IBGE, 2019).

En Ilha Solteira, municipio de este estudio, uno de los temas ambientales más relevantes es la recolección selectiva. No existe una estructura organizada para dicha colección, que genera una colección privada por parte de los recolectores, además de causar un almacenamiento inadecuado, causar mal olory, a menudo, atraer animales sinantrópicos, transmisores de varios patógenos (RITA et al, 2017).

Por lo tanto, el objetivo de este estudio fue evaluar los perfiles socioeconómicos, demográficos y laborales de los recicladores reciclables de Ilha Solteira, São Paulo, y analizar la importancia de esta práctica para la subsistencia y la preservación del medio ambiente. 


\section{Metodología}

Este estudio se realizó en Ilha Solteira, ubicada en el noroeste del estado de São Paulo. El método de investigación utilizado fue cuantitativo y cualitativo, también llamado encuesta de campo, así como visitas técnicas y entrevistas con los recolectores de material reciclable.

Veintidós fueron entrevistados a los recolectores de material reciclable de este municipio durante el período comprendido entre diciembre de 2018 y febrero de 2019. Se les preguntó sobre temas relacionados con su actividad y su importancia para su subsistencia.

El cuestionario constaba de siete preguntas que cubrían temas relacionados con los datos demográficos sociales del recolector, por qué trabajan en esta área, su relación en el trabajo, su percepción de los problemas ambientales, la composición del ingreso, las políticas compensatorias y sus sentimientos sobre la profesión que tienen. ejercicios

También se recopiló información del Ayuntamiento de Ilha Solteira. En una reunión con el alcalde y los secretarios municipales de los sectores involucrados (Departamento de Obras, Salud, Desarrollo Social y Departamento Legal), se presentó el proyecto. Hubo algunas discusiones sobre temas relacionados con la importancia de la recolección selectiva para la reducción y reutilización de los desechos generados por el municipio, y consultas en el sitio web del municipio.

Los datos recopilados se tabularon en las hojas de cálculo del software Microsoft Office Excel para formar la base de datos. Luego, se analizaron calculando medias simples y porcentajes.

En cuanto a los aspectos éticos, el proyecto fue presentado al Comité de Ética de Investigación de la Universidade Brasil. Comenzó después de la aprobación con el número de protocolo 2.867.037. La participación individual se produjo después de firmar el formulario de consentimiento informado. 


\section{Resultados y Discusión}

Esta investigación demuestra el perfil de los recicladores en el municipio de Ilha Solteira, basado en variables sociodemográficas como el género, el estado civil, las condiciones de vida, la duración del trabajo en el área y la educación. Se analizaron otros indicadores sobre motivaciones y condiciones de trabajo, inseguridad laboral, ingresos y discriminación.

Según Bortoli (2009), en Brasil, la profesión de reciclador fue reconocida y oficializada en 2002 por la Clasificación Brasileña de Ocupaciones (CBO).

Sin embargo, se estima que hay más de 500,000 recicladores en el país (REIS et al, 2016), sin embargo:

[...] El reconocimiento de la profesión no implicó un cambio en las condiciones de vida y de trabajo de los recolectores, quienes trabajan sin empleo y sin derechos, generalmente ganan menos de un salário mínimo, disputan materiales reciclables con sus compañeros, no se insertan en gestión de residuos y enfrentar la explotación de la industria del reciclaje. (BORTOLI, 2009, p. 107).

Identificamos 22 recicladores que trabajan en Ilha Solteira. De estos, el 72.7\% eran hombres. Un estudio realizado en el estado de Minas Gerais encontró que el 50.5\% de los recolectores eran hombres (FREITAS; FERREIRA, 2015). En otros estudios, se encontraron datos encontrados opuestos a esta investigación. Ipameri, GO, tenía el $71.4 \%$ de las recicladoras en la ciudad de Río de Janeiro, el 62.5\% también eran mujeres, así como el $56.7 \%$ de los recicladores que trabajan en el Parque São José en Fortaleza y 62.1 \% en Brasilia, DF (RIBEIRO et al., 2009; GONÇALVES et al, 2013; MOREIRA, 2013; CRUVINEL et al., 2017).

En cuanto al estado civil, el $54.5 \%$ de los recolectores que trabajan en llha Solteira declararon estar casados, seguidos por el $27.3 \%$ solteros y el $18.2 \%$ separados y / o viudos. Una encuesta realizada por Soares (2014) en Ceilândia encontró que la mayoría (64.6\%) de los recolectores eran solteros, al igual que Cruvinel et al (2017), quienes evaluaron dos grupos de recolectores y descubrieron que $67.8 \%$ y $65,6 \%$ también eran solteros. El hecho 
de que Ilha Solteira tenga la mayoría de los recicladores casados y varones puede estar relacionado con la necesidad del jefe de familia de mantener la casa, y estar desempleado, el trabajo con el reciclaje fue la única opción encontrada.

Un punto importante a tener en cuenta es que en algunos estudios se observaron muchos recicladores con vínculos de parentesco. Estos lazos entre los miembros dificultan que las personas nuevas se unan a la actividad que no tienen relación familiar (RIBEIRO et. Al., 2009; GONÇALVES et al, 2013; MOREIRA, 2013).

En términos de educación, el 50\% de los coleccionistas tenían escuela primaria, el $31.8 \%$ escuela secundaria y el $18.2 \%$ analfabetos. En otras investigaciones, se encontraron datos similares, como RIBEIRO et al. (2009), donde el 75\% de los coleccionistas tenían escuela primaria, Cruvinel et al. (2017), 36.5 y 33.2\% y Neves et al. (2017) reportaron 87.2\% con la escuela primaria. La baja escolaridad es una razón importante para adherirse a la práctica de recolectar material reciclable. Freitas y Ferreira (2015) en su investigación también declararon que los recolectores tenían una visión clara sobre el hecho de que la escolarización influye en las condiciones de empleabilidad.

Muchos coleccionistas no terminan sus estudios por várias razones, incluida la falta de aliento, la necesidad de ayuda para apoyar a sus familias, así como los analfabetos informan las mismas razones y la mayoría se siente lo suficientemente mayor como para regresar a las actividades escolares (SANTOS et al. al., 2018).

La escolarización es un factor muy importante con respecto a la inserción en el mercado laboral. Según datos de IPEA (2013), la tasa de analfabetismo entre los recicladores alcanza aproximadamente el $20,5 \%$ de las personas. Este hecho demuestra el grave problema social que enfrentan estas personas, ya que una persona analfabeta se enfrenta a grandes limitaciones con respecto a las oportunidades profesionales.

En cuanto a las condiciones de vivienda, el 54,6\% de los recolectores viven "a favor" en las casas de amigos o familiares. Esta situación se repite en otras encuestas, donde la mayoría de los encuestados vivía a favor o pagaba el alquiler. Además, los declarantes también informaron que vivían en condiciones a menudo poco saludables (RIBEIRO et. Al., 
2009; MOREIRA, 2013). En Ceilândia, el 56,6\% de los recolectores tenían su propia casa (SOARES, 2014).

A pesar de que la recolección selectiva es un mercado cada vez más prometedor debido a la creciente preocupación por los problemas ambientales, y que puede generar ingresos, el trabajo de recolección todavía está relacionado con condiciones de marginalidad y falta de derechos para los recolectores, partes fundamentales para esto. sector. Incluso participando como elementos básicos para el sector, trabajan en condiciones precarias, sin condiciones de una vida digna (LEAL et al., 2002). Por lo tanto, debe tenerse en cuenta que la atención de las agencias públicas a los recicladores debe revisarse con respecto a la formalización de la profesión y la creación de cooperativas destinadas a fortalecer la clase que actualmente está tan excluida de la sociedad (SANTOS et al., 2018)

Con respecto al tiempo de trabajo en esta industria, el 40.9\% informó haber recolectado material reciclable entre 5 y 10 años, el $31.8 \%$ entre 1 y 5 años y el $23.7 \%$ durante 10 años. Estos datos corroboran la investigación realizada en Brasilia, donde en promedio el $52.4 \%$ de los recicladores trabajaron durante 5 a 10 años en la actividad y el $23.9 \%$ entre 1 a 5 años (CRUVINEL et al., 2017). Neves et al. (2017), en una encuesta realizada en Chapecó, SC, encontró que el 38\% de estos profesionales habían estado trabajando en el campo durante más de 10 años. Estos datos demuestran que la profesión de carroñero no es temporal y termina siendo consolidada, porque los trabajadores usan la práctica durante muchos años, ya sea para complementar el ingreso familiar o incluso por falta de oferta de trabajo.

Cuando se le preguntó por qué el individuo se había unido a la profesión de carroñero, los resultados se describen en la Figura 1

(Continue...) 
Figura 1 - Razón por la cual el individuo se unió a la profesión de reciclador en llha Solteira, SP, 2019

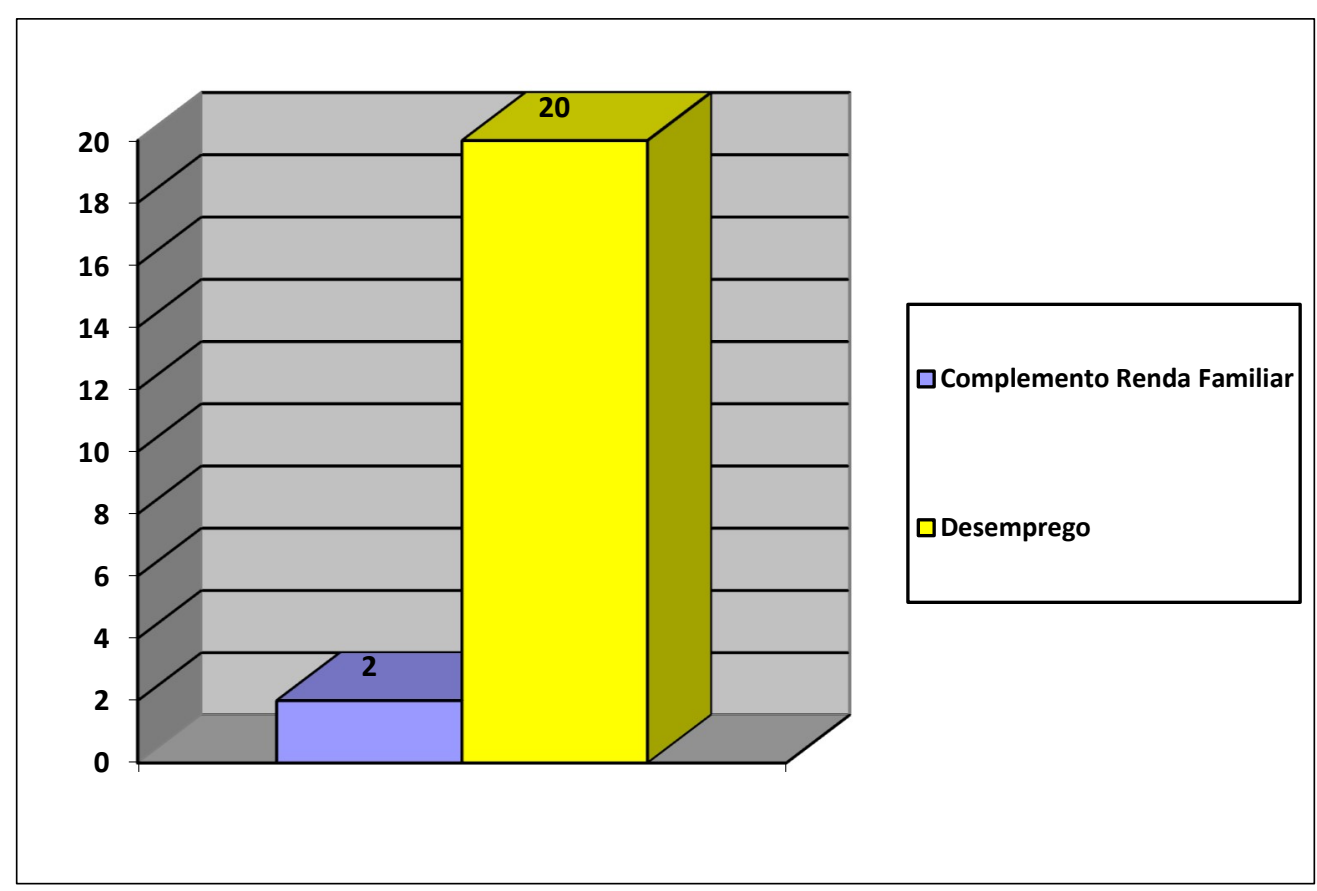

Según los encuestados en esta encuesta, el 90.9\% declaró que comenzaron a trabajar como recicladores debido a su condición de desempleados. Freitas y Ferreira (2015), también señalaron el desempleo como la principal motivación para la adhesión a esta profesión, y también informaron la necesidad de complementar el ingreso familiar. Otros autores también informaron en su investigación el desempleo como el factor principal de la adherencia de la recolección de material reciclable (RIBEIRO et. Al., 2009; GONÇALVES et al., 2013; MOREIRA, 2013). La actividad laboral de estos trabajadores termina siendo una forma de supervivencia y eludir la falta de oportunidades de trabajo formal, por lo que están motivados únicamente por la necesidad de supervivencia.

En una investigación de Gonçalves et al. (2013) los recicladores dijeron que se unieron a la actividad debido a la falta de oportunidades. En el pasado, los recicladores trabajaban como sirvientas, granjeras, costureras, albañiles y amas de casa. El desempleo, la necesidad de mantener a sus hijos o la enfermedad de parientes mayores se mencionaron como causas del comienzo de la actividad como carroñero. En los discursos de los recolectores, la falta 
de oportunidades es un elemento fundamental para dirigir o mantener a las personas en esta actividad.

Otro tema analizado fue la relación del recolector en su entorno de trabajo. En llha Solteira, el $81.8 \%$ dijo que tenía una gran relación con otros colegas, el 9.1\% dijo que tenía una buena relación y el 9\% dijo que tenía una mala relación. El hecho de que la mayoría informe excelentes y buenas relaciones con sus colegas puede estar relacionado con el problema ya abordado por Gonçalves et al. (2013) cuando declararon en su investigación que los recolectores tienen vínculos de parentesco entre ellos, lo que garantiza una división razonable de las ganancias.

También se investigaron cuestiones ambientales a través de entrevistas con recolectores de material reciclable de Illha Solteira. Entre los encuestados, el $66.3 \%$ declaró que considera que la práctica de la recolección es muy importante para mantener una ciudad más limpia y para reducir la contaminación del agua.

Es bien sabido que el reciclaje tiene varios beneficios para el medio ambiente, ya que preserva los recursos naturales, reduce la contaminación del suelo, el agua y el aire, mejora la limpieza urbana y genera ingresos (LOMASSO et al., 2015).

Siguiendo la misma línea de pensamiento, el 90.9\% dijo que consideraba su acción extremadamente importante para minimizar la contaminación visual de la ciudad y reducir la cantidad de desechos enviados a los vertederos, así como para preservar el medio ambiente. Ribeiro et al., 2009, observaron que los entrevistados demostraron su percepción de la interacción de su proceso de trabajo con el medio ambiente, lo que se puede notar en el discurso de los recolectores: "Soy una persona que cuida el medio ambiente". O "La reducción de residuos mejora la salud y es un patrimonio para las generaciones futuras".

En una investigación realizada en Ceará, los trabajadores en esta área son una solución barata al problema de los desechos en las ciudades. Además, los recicladores han desarrollado una actividad que es necesaria para el mundo de consumo actual, el gobierno, los negocios y la sociedad. Todos estos sectores generan y descartan la basura, pero olviden que en la naturaleza no se pierde nada, pero todo se transforma (MOREIRA, 2013). 
Con respecto a la satisfacción con la profesión, todos los recolectores informaron haber realizado el cambio de trabajo si había alguna oportunidad, debido a que habían sufrido algún tipo de prejuicio debido a su trabajo.

El mercado laboral es el lugar donde los prejuicios se manifiestan de las formas más perversas, especialmente cuando se trata de la profesión de reciclador (DURHAM, 2013). Esta profesión expone al individuo a los males que provienen de la basura, como el riesgo de contagio de enfermedades, falta de seguridad, además de sufrir prejuicios y discriminación debido a su profesión poco saludable (MOREIRA, 2013).

Aunque es un trabajo poco saludable, todos los recicladores de llha Solteira declararon que estaban satisfechos con su situación actual. Esto se debe a que es mejor para ellos tener una fuente de ingresos para mantener a sus familias que estar desempleados. Estos datos son similares a la investigación de Gonçalves et al. (2013), donde la representación social más común encontrada entre los encuestados es que preferían este trabajo al robo, la trata, la mendicidad, lo que sin querer refuerza su significado de miseria y exclusión, y también la autoimagen de estas personas. como sin otra alternativa que "vivir de la basura".

Ribeiro et al. (2009), observaron que con respecto a las condiciones de trabajo, era unánime el deseo de mejores días reportados por los recicladores, ya que consideran una actividad repetitiva, exhaustiva, peligrosa y poco saludable. Los encuestados a menudo señalaron la necesidad de habilidad manual, concentración, atención y responsabilidad. También dijeron que esta actividad profesional implica, entre otros factores, una postura incorrecta, movimientos repetitivos, esfuerzo visual e implica el uso de la fuerza. Los riesgos percibidos por los trabajadores son los de accidentes que a menudo ocurren con objetos punzantes y enfermedades transportadas por los desechos que se llevan al cribado y por vectores como ratas, cucarachas y mosquitos.

La Tabla 1 proporciona información sobre los ingresos obtenidos por los recicladores al final del mes, donde, en Ilha Solteira, el ingreso promedio del 60,3\% de los encuestados está entre $R \$ 301,00$ a $R \$ 500,00$ reales. 
Tabla 1 - Composición de ingresos por mes de recicladores reciclables de Ilha Solteira, SP

\begin{tabular}{lc}
\hline \multicolumn{1}{c}{ Ingresos } & Número de recolectores \\
\hline Hasta $R \$ 100$ & 1 \\
\hline$R \$ 101$ a $R \$ 300$ & 3 \\
\hline$R \$ 301$ a $R \$ 500$ & 14 \\
\hline$R \$ 501$ a $R \$ 1000$ & 4 \\
\hline Encima de $R \$ 1000$ & 0 \\
\hline
\end{tabular}

Datos similares fueron encontrados por Santos et al. (2018) en Três Passos, RS, donde el 53,3\% informó ingresos entre $\mathrm{R} \$ 100,00$ a $\mathrm{R} \$ 400,00$. En Ipameri, el ingreso mensual individual promedio obtenido en la actividad es de R \$ 670.00 (GONÇALVES et al., 2013).

La investigación realizada en el estado de Minas Gerais mostró que el ingreso promedio de un recolector de material reciclable que trabaja fuera del vertedero se estima en $\mathrm{R} \$ 570,19$, mientras que el ingreso de un recolector que trabaja directamente en el vertedero es ligeramente mayor, en promedio. R \$615,27. En Chapecó, SC, solo el 15\% de los recicladores entrevistados informaron ingresos por debajo de R \$ 500.00 (NEVES et al., 2017).

Varios factores pueden estar relacionados con los ingresos obtenidos de la actividad, entre ellos podemos mencionar el tipo de material recolectado, porque su venta está relacionada con la lista de precios; la edad del coleccionista; El tiempo disponible para realizar la actividad y los problemas de salud (SANTOS et al., 2018).

Se preguntó a los recolectores entrevistados en esta encuesta si reciben algún beneficio social del Gobierno Federal. En Ilha Solteira, 14 de los encuestados reciben una canasta básica de alimentos y 8 reciben la asignación familiar.

Una investigación realizada en Brasilia mostró que, en promedio, el 24,3\% de los recicladores entrevistados recibió una asignación familiar (CRUVINEL et al., 2017). En Fortaleza, el 12.5\% informó haber recibido el beneficio (MOREIRA, 2013). Los programas de 
asistencia del gobierno son extremadamente importantes para ayudar a mejorar las vidas de los ciudadanos de bajos ingresos. El Programa Bolsa Familia, por ejemplo, es una modalidad de transferencia de asistencia social relacionada con los derechos sociales como la salud y la educación, que beneficia a miles de familias pobres y extremadamente pobres (WEISSHEIMER, 2006).

Con respecto a los datos obtenidos del Ayuntamiento de llha Solteira sobre el destino de los desechos generados y la recolección selectiva de la ciudad, así como los programas de concientización sobre la importancia del destino correcto de los desechos y el reciclaje, se encontró que, en su sitio web, El Ayuntamiento proporciona una gran cantidad de información sobre cómo los ciudadanos pueden ayudar en la recolección selectiva y el control de los desechos generados.

La primera información obtenida fue una campaña llamada "Ciudad más limpia".

El objetivo de esta campaña era concienciar a la población de su papel en el mantenimiento de la limpieza pública demostrando que cada ciudadano puede tomar medidas simples para mejorar su entorno de vida. Acciones como esta contribuyen en gran medida a la preservación del medio ambiente, ya que cualquier cantidad de desechos que no se viertan ilegalmente en el medio ambiente ya será un gran logro, por lo que la conciencia del público sobre la importancia de preservar el medio ambiente a través de la reducción del vertido. de residuos contaminantes es muy importante (SOARES, 2014).

Una forma económica y efectiva de actuar como recicladores es la organización a través de cooperativas (NASCIMENTO, 2000). Las cooperativas tienen su objetivo centrado en los aspectos sociales y económicos, que apuntan principalmente a la distribución del ingreso y la inclusión social (SOARES, 2014).

En Ilha Solteira, existe una cooperativa de reciclaje selectivo de recolección (Figura 2) en la cual el ayuntamiento ofrece apoyo. 
Figura 2 - Cooperativa de reciclaje de Ilha Solteira, São Paulo

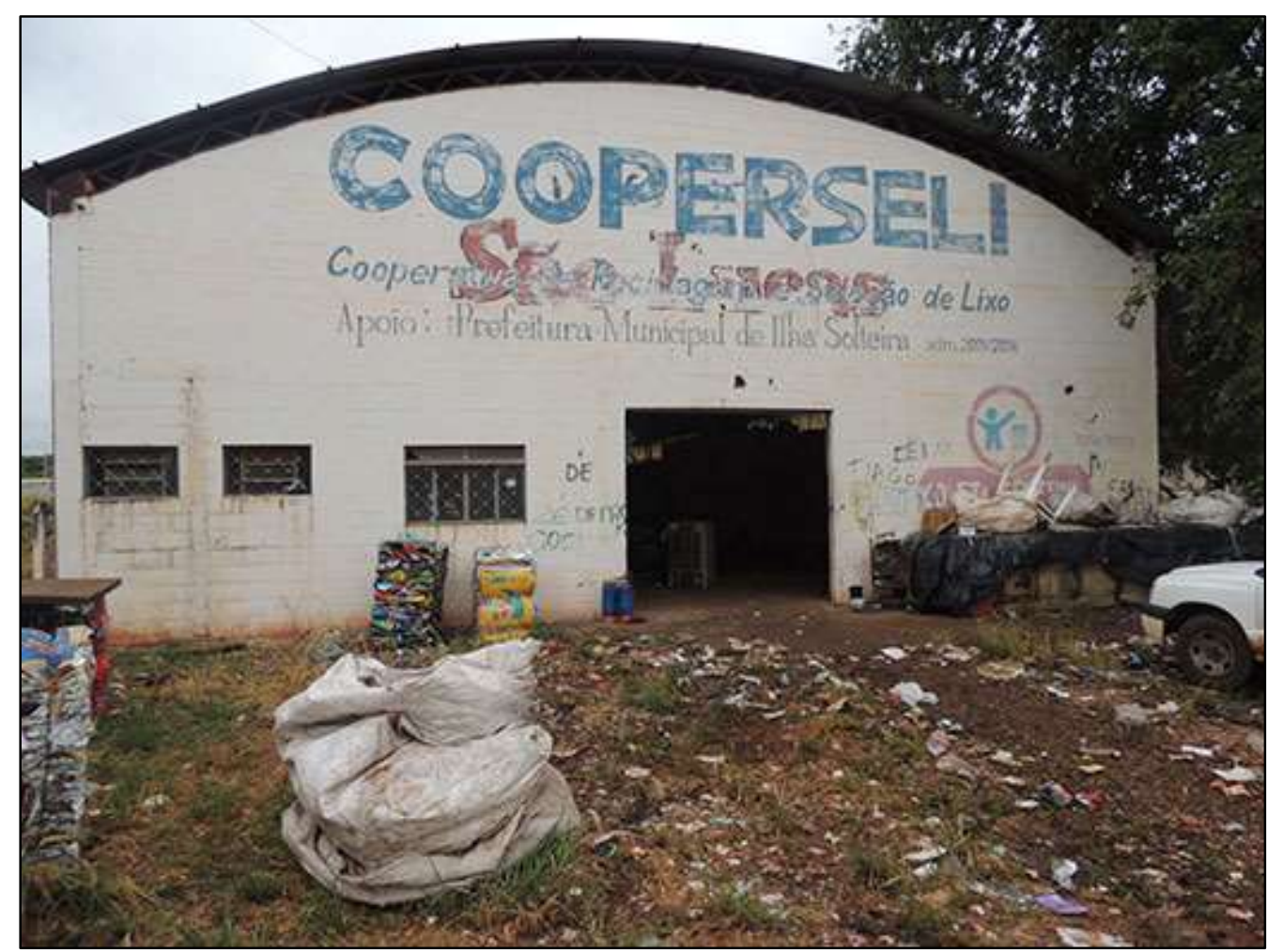

Fuente - Banco de Noticias de la Ciudad de Ilha Solteira, 2016

En 2016, el Ayuntamiento de Ilha Solteira, con el objetivo de resolver problemas relacionados con la recolección selectiva de la ciudad, revitalizó la cooperativa de reciclaje, según las noticias:

El martes 10 de mayo, el Municipio de llha Solteira Tourist Resort envió un equipo de profesionales de los sectores del Ayuntamiento para realizar el mantenimiento, la limpieza y la revitalización de la Colección Cooperativa de Reciclaje (COOPERSELI).

El Ayuntamiento lleva mucho tiempo estudiando una forma de resolver los problemas de la recogida selectiva, ya que cuanto menos materiales reciclables lleguen al vertedero, mejor evitaría un mayor costo en la construcción de nuevas celdas de vertedero que causarían daños considerables a la caja flerte Público.

La implementación de la recolección selectiva en 2002, y la creación de la cooperativa de reciclaje y poco después de la construcción del Relleno Sanitario Municipal, fue importante considerando que con menos desechos reciclables enviados al mejor Relleno Sanitario! Esto aumenta la capacidad del vertedero. 
Con el tiempo, con el aumento del consumismo de productos y materiales por parte de los isleños, se produjo una mayor disposición de materiales reciclables, lo que generó una mayor demanda e incluso con las campañas llevadas a cabo por la ciudad de orientación de separación de reciclables los miércoles. No fue posible para la población en general reciclar mejor sus desechos.

En Ilha Solteira, los desechos reciclables mezclados con los restos orgánicos se depositan en el Relleno Sanitario, dejando mucho que desear.

La falta de apoyo e inversión en el sector también llevó a la cooperativa a caminar lentamente, llegando al punto de crisis e incapaz de caminar.

Debido a las limitaciones de la productividad y la recolección individual de residuos sólidos reciclables por parte de los recicladores, es extremadamente importante crear programas de reciclaje destinados a la creación de empresas, que son asociaciones o cooperativas, por lo tanto, el volumen de material enviado a las industrias de reciclaje. sería más grande, generando ahorros e ingresos en todo el sector (FREITAS; FERREIRA, 2015).

En los últimos años, se han creado varias cooperativas y asociaciones de recicladores, así como varios gobiernos locales han instituido programas de recolección selectiva en municipios, incluidos los recicladores autónomos (RIBEIRO, et al., 2014). Este hecho es muy importante porque entre las ventajas de las cooperativas de recolectores podemos mencionar la generación de empleos e ingresos; la recuperación de la ciudadanía de estos recolectores, ya que la mayoría de ellos no tienen hogar; selector de protección de la salud; y organización del trabajo realizado por el coleccionista, entre otros (SANTOS et al., 2018).

Cuando analizamos toda la cadena relacionada con el reciclaje de materiales, el recolector se considera el pilar de la actividad. Esto se debe a que lo mismo es quién lleva el material a las calles, lo reenvía a la cooperativa o las asociaciones, haciendo que este material llegue a la industria del reciclaje y pueda ser utilizado nuevamente. Sin embargo, también se considera el eslabón más débil de esta cadena, ya que integra una categoría de trabajadores en la que no tienen organización colectiva (MONTENEGRO, 2001).

Para revertir la situación relacionada con la falta de organización colectiva de esta clase, es necesario sensibilizar a los recicladores para que puedan organizarse políticamente, 
porque solo entonces pueden luchar por sus derechos y finalmente ver nuevos horizontes capaces de generar una mejor calidad de vida y de trabajo (MOREIRA, 2013).

Después de analizar los datos sobre la situación de los recicladores en la ciudad de Ilha Solteira, São Paulo, se desarrolló material de apoyo instructivo en formato de carpeta. Este material se distribuyó a la población y contiene información relacionada con la importancia de la práctica del reciclaje en relación con la preservación del medio ambiente y como fuente de empleo y generación de ingresos para varias personas necesitadas.

\section{Conclusión}

La profesión de reciclador ha demostrado ser muy importante, lo que se puede comparar en otros estudios que utilizan esta práctica como ocupación y fuente de ingresos para mantener a sus familias, incluso si la práctica no es saludable cuando se realiza de forma autónoma. Por lo tanto, la colección debe realizarse de manera digna y tener el debido reconocimiento y reconocimiento por parte de la sociedad debido a la gran importancia también con respecto a los problemas ambientales.

La necesidad de cambios relacionados con las políticas públicas era evidente, ya que los recolectores no tienen los recursos financieros para mejorar su trabajo y su entorno familiar por sí solos. Ante esto, es necesario el empoderamiento de los recicladores con el objetivo de su inclusión en el entorno social, para que la sociedad vea la importancia de este profesional para el medio ambiente.

El trabajo cuando se realiza de manera cooperativa es muy positivo, pero aún se encuentra con resistencia. Por lo tanto, la capacitación de los recicladores, la seguridad laboral y la prevención de enfermedades asociadas con la actividad deberían discutirse mejor con esta clase trabajadora, así como las prácticas de educación ambiental deberían desarrollarse con la población con respecto a la separación del material reciclable. La inclusión social y la adición de valor a los residuos reciclados también pueden ser alternativas para mejorar los ingresos y la calidad de vida de los trabajadores. 


\section{Agradecimientos}

Universidade Brasil, campus de Fernandópolis

Prefeitura Municipal de llha Solteira

\section{Referencias}

BORTOLI, M. A. Catadores de materiais recicláveis: a construção de novos sujeitos políticos. Revista Katálysis, Florianópolis: Edufsc, v. 12, n.1, p. 105-114, jan./jun. 2009. Disponível em: <http://www.scielo.br/pdf/rk/v12n1/13.pdf>. Acesso em: 10 abr. 2019;

BRASIL. Instituto Brasileiro de Geografia e Estatística. Características Gerais dos Domicílios e dos Moradores da Pesquisa Nacional por Amostra de Domicílios Contínua 2018. Disponível em: <https://agenciadenoticias.ibge.gov.br/agencia-sala-de-imprensa>. Acesso em 18 de abr. De 2019.

CRUVINEL, V. et al. Perfil dos Catadores de Resíduos Sólidos do Distrito Federal: Uma Análise Comparativa entre Associações de Ceilândia e Estrutural. [Artigo]. Hegemonia - Revista Eletrônica de Relações Internacionais do Centro Universitário. UNIEURO, Brasília, número 19, Janeiro-Junho de 2017.2 Acesso em: <file:///D:/Documents/Downloads/cruvinel\%20et\%20al\%202017.pdf>. Acesso em 08 abr. 2019.

DURHAM, E. R. Desigualdade educacional e quotas para negros nas universidades. Núcleo de Pesquisas sobre Ensino Superior da Universidade de São Paulo, 2013.

FREITAS, D. G. DE. FERREIRA, F. P. M. et al. Perfil dos Catadores de Materiais Recicláveis nos Lixões de Minas Gerais. Caderno de Geografia, vol. 25, núm. 44, julho-dezembro, Pontifícia Universidade Católica de Minas Gerais. Belo Horizonte ,2015.

GONÇALVES, C. V.et al. A vida no lixo: um estudo de caso sobre os catadores de materias recicláveis no município de Ipameri (GO). Editora Holos, Ano 29, v. 2., Ribeirão Preto, 2013.

IPEA- Instituto de Pesquisa Econômica Aplicada. Situação Social dos catadores e catadoras de material reciclável e reutilizável. Brasília: IPEA, 2013.

Lago, A. A. C. do. Estocolmo, Rio de Janeiro, Johanesburgo: O Brasil e as Três Conferências Ambientais das Nações Unidas. Brasília: Instituto Rio Branco, Fundação Alexandre de Gusmão - FUNAG, 2007.

LEAL, A. C. et al. A reinserção do lixo na sociedade do capital: uma contribuição ao entendimento do trabalho na catação e na reciclagem. Revista Terra Livre, São Paulo, ano 18, n. 19, p. 177-190, jul/dez, 2002. 
LIMA, A. E. F.et al.Educação Ambiental: O Lixo em Questão, 2007. Disponível em: $<$ http://www.guiabioagri.com.br. Acesso em 07 abr.2019.

LOMASSO, A. L. M. Benefícios e desafios na implantação da reciclagem: um estudo de caso no Centro Mineiro de Referência em Resíduos (CMRR). Revista Pensar Gestão e Administração, v. 3, n. 2, jan. 2015.

MONTENEGRO, D. M. Trabalho, lixo e lucro: precariedade do trabalho no circuito econômico da reciclagem. In: Anais do XI Congresso Luso Afro Brasileiro de Ciências Sociais: diversidades e (des)igualdades. Salvador. Universidade Federal da Bahia, 2011.

MARODIM, V. S, MORAIS, G. A. Educação Ambiental com os temas geradores lixo e água e a confecção de papel reciclável artesanal. Anais do $2^{\circ}$ Congresso Brasileiro de Extensão Universitária. Belo Horizonte.Disponível em: www.ufmg.br/congrext/educa/.Acesso em 18 de abr. de 2019.

MOREIRA, A. T. X. (Sobre) vivendo do lixo: um estudo das condições de trabalho e de vida dos catadores de lixo do Bairro Parque São José.[Monografia]. Bacharelado em Serviço Social. Centro de Ensino Superior do Ceará Faculdade Cearense. Fortaleza, 2013.

NASCIMENTO, F. R. Cooperativismo como alternativa de mudança: uma abordagem normativa. Rio de Janeiro: Forense, 2000.

NEVES, L. M. Catadores de materiais recicláveis: perfil social e riscos à saúde associados ao trabalho. Revista Brasileira de Geografia Médica e da Saúde. Disponível em: < http://www.seer.ufu.br/index.php/hygeia>. Acesso em 08 abr. 2019.

PREFEITURA DE ILHA SOLTEIRA. Coleta de Lixo. 2018. Disponível em: <http://www.lhasolteira.sp.gov.br/index.php/noticias/93 >. Acesso em: 08 abr. 2019.

PREFEITURA DE ILHA SOLTEIRA. 2018. Disponível em: <http://www.llhasolteira.sp.gov.br/index.php/noticias/93>. Acesso em: 01 abr. 2018.

REIS, I. R. Catadores: as histórias por trás da reciclagem. Intercom - Sociedade Brasileira de Estudos Interdisciplinares da Comunicação XXIII Prêmio Expocom 2016 - Exposição da Pesquisa Experimental em Comunicação. Universidade Federal de Uberlândia - MG

RIBEIRO, J. C. DE F. Et al. Catadores de materiais recicláveis: estudo de caso de uma cooperativa na cidade do Rio de Janeiro. V Congresso Nacional de Excelência em Gestão. Gestão do Conhecimento para a Sustentabilidade. Niterói, 2009

RITA, F. DOS S. et al. Promoção da saúde: o papel da enfermagem no controle de animais sinatrópicos no correto armazenamento de resíduos. $14^{\circ}$ Congresso Nacional de Meio Ambiente. Poços de Caldas, de 26 a 29 de setembro de 2017.

SALDIVA, PAULO. Meio ambiente e saúde: o desafio das metrópoles. São Paulo: ExLibris Comunicação Integrada, 2010. 
Santos, C. dos. Perfil socioeconomico de catadores de materiais recicláveis do Municipio de Três Passos (RS). Revista Extensão em Foco, nº 15, Jan/ Jul, p.56 - 70, 2018.

SÃO PAULO. Divisão de Comunicação. Prefeitura de Ilha Solteira. Prefeitura de llha Solteira dá apoio a Cooperseli. 2016. Disponível em: <http://www.ilhasolteira.sp.gov.br/bancodenoticias/view.php?id=3262\&pag=4>. Acesso em: 11 abr. 2019.

SOARES, D. L. C. Análise dos riscos ocupacionais e acidentes de trabalho em catadores de resíduos sólidos em Cooperativas de Ceilândia - DF. [TCC]. Bacharelado em Saúde Coletiva. Universidade de Brasília - UnB, Faculdade de Ceilândia - Fce. Ceilândia, 2014.

WEISSHEIMER, M. A. Bolsa família: avanços, limites e possibilidades do programa que está transformando a vida de milhões de famílias no Brasil. São Paulo: Fundação Perseu Abramo, 2006.

WEYERMÜLLER, A. R. Direito Ambiental e Aquecimento Global. São Paulo: Atlas, 2010. 